University of Nebraska - Lincoln

DigitalCommons@University of Nebraska - Lincoln

Faculty Publications, Department of Physics and Astronomy

Research Papers in Physics and Astronomy

8-1-2005

\title{
PHOTOMETRY OF TYPE II CEPHEIDS. III. THE INTERMEDIATE- PERIOD STARS
}

\author{
Edward G. Schmidt \\ University of Nebraska-Lincoln, eschmidt1@unl.edu \\ Dale Johnston \\ University of Nebraska-Lincoln, dalej96@gmail.com \\ Shawn Langan \\ University of Nebraska-Lincoln, nuaur@hotmail.com \\ Kevin M. Lee \\ University of Nebraska-Lincoln, klee6@unl.edu
}

Follow this and additional works at: https://digitalcommons.unl.edu/physicsfacpub

Part of the Physics Commons

Schmidt, Edward G.; Johnston, Dale; Langan, Shawn; and Lee, Kevin M., "PHOTOMETRY OF TYPE II CEPHEIDS. III. THE INTERMEDIATE-PERIOD STARS" (2005). Faculty Publications, Department of Physics and Astronomy. 23.

https://digitalcommons.unl.edu/physicsfacpub/23

This Article is brought to you for free and open access by the Research Papers in Physics and Astronomy at DigitalCommons@University of Nebraska - Lincoln. It has been accepted for inclusion in Faculty Publications, Department of Physics and Astronomy by an authorized administrator of DigitalCommons@University of Nebraska - Lincoln. 


\title{
PHOTOMETRY OF TYPE II CEPHEIDS. III. THE INTERMEDIATE-PERIOD STARS
}

\author{
Edward G. Schmidt, Dale Johnston, Shawn Langan, and Kevin M. Lee \\ Department of Physics and Astronomy, University of Nebraska, Lincoln, NE 68588-0111; \\ eschmidt1@unl.edu,dalej96@bigred.unl.edu,nuaur@hotmail.com,klee6@unl.edu \\ Received 2005 April 4; accepted 2005 April 13
}

\begin{abstract}
We present 741 new photometric observations for 22 Cepheids with periods between 3 and 8 days. Many of the stars are probable type II Cepheids, but we have included some type I Cepheids for comparison. The shapes and stability of the shapes of the light curves, the stability of the periods, and the scatter of individual data points are discussed. Although none of these proves to be a reliable discriminator between type I and type II Cepheids, they do permit the identification of several unusual stars.
\end{abstract}

Key words: Cepheids - stars: Population II

Online material: machine-readable tables

\section{INTRODUCTION}

This is the final paper of three that present and discuss photometric observations of field type II Cepheids. Schmidt et al. (2004a, hereafter Paper I) and Schmidt et al. (2005, hereafter Paper II) considered long-period ( $P>8$ days) and short-period ( $P<3$ days) stars, respectively, while the present paper is concerned with stars that fall between the two groups.

While it is easy to distinguish type II from type I Cepheids when they occur in clusters, the distinction is often ambiguous for individual field stars. We have undertaken a study aimed at developing reliable observational criteria to identify type II Cepheids in the field. The present series of papers and another that discussed the spectra of type II Cepheids (Schmidt et al. 2003a, 2003b, 2004 b) provide the observational basis for this project. The reader should refer to Paper I for a more extended discussion of this project.

The General Catalogue of Variable Stars (Kholopov 1985, 1987; hereafter the GCVS) lists 42 stars with periods less than 3 days and 113 with periods greater than 8 days that are classified as type II Cepheids (GCVS classes CW, CWA, or CWB). By comparison, only 17 such stars fall between these periods.

This bimodal period distribution is attributed to the different evolutionary stages of the short- and long-period stars (Gingold 1976). The short-period group is identified with asymptotic giant branch stars evolving back into the instability strip in response to adjustments between the hydrogen- and helium-burning shells. The long-period group is identified with a later stage of evolution in which second giant branch stars undergo blue loops due to helium flashes or are evolving blueward for a final time when the hydrogen-burning shell approaches the surface of the star. However, uncertainties remain in that the frequency distribution with luminosity predicted by the models does not agree with observation. Thus, the occurrence of type II Cepheids in the intermediateperiod range can potentially constrain the evolutionary models.

There have been a number of studies of the light curves of Cepheids that included stars in this period range. Among these are Fourier decomposition studies by Simon \& Moffett (1985), Antonello \& Poretti (1986), Andreasen (1988), Antonello et al. (1990), Mantegazza \& Poretti (1992), Fernie \& Ehlers (1999), and Zakrzewski et al. (2000). However, virtually none of the intermediate-period stars in these studies are classified as type II Cepheids in the GCVS or appear in Harris's (1985) catalogue of likely type II Cepheids. The present study addresses this shortcoming by including 12 intermediate-period stars that are listed as type II Cepheids in either of these catalogs. We have also included a number of type I stars and stars of indeterminate type (CEP in the GCVS) for comparison.

\section{THE OBSERVATIONS}

The observations were all made at Behlen Observatory with the $0.76 \mathrm{~m}$ telescope. The instrumentation and the procedures used in collecting and processing the data are the same as for the observations presented in Paper I, and the reader is referred to that publication for a complete description of them.

The stars discussed here are listed in Table 1, where column (1) lists the names of the stars, column (2) lists their approximate periods, and column (3) gives the classifications from the GCVS. A "II" in column (4) identifies stars that appear in Table 1 of Harris's (1985) catalog of type II Cepheids. He selected these stars on the basis of $|Z|$, the distance from the Galactic plane, so we follow Papers I and II in referring to them as high- $Z$ stars. Stars without an entry in column (4) are referred to as low- $Z$ stars. While this distinction is useful in our discussion, we should bear in mind that it is at best a rough guide to type for individual stars. Information about the comparison stars is provided in columns (5), (6), and (7). Column (5) lists the number of comparison stars used for each field. Column (6) contains the standard errors of the adopted $V$ and $R$ magnitudes of the comparison stars. Column (7) gives the number of photometric nights included in determining the comparison star magnitudes.

As discussed in Paper I, changes in the instrumentation over the span of the observations resulted in the adoption of different sets of comparison stars for some variables. Such cases are reflected in Table 2.

Because Behlen Observatory is a low-quality photometric site, placing the comparison stars on the standard system presents difficulties. As a check, we compared our $V$ light curves with those from earlier photometry (references in Table 1, col. [10]). In a few cases, small zero-point shifts were apparent, and we adjusted our photometry to match the earlier photometry. These are identified by a footnote to the table. Unfortunately, no such check is possible for the $R$ magnitudes.

In column (8) of Table 1 we list the range of Julian Dates of our observations. In our analysis we have incorporated $V$ photometry from the literature for most of the stars in addition to our new observations. In column (9), the first number is the total number of light-curve points available, while the number in parentheses 
TABLE 1

The Program Stars

\begin{tabular}{|c|c|c|c|c|c|c|c|c|c|}
\hline $\begin{array}{l}\text { Star } \\
(1)\end{array}$ & $\begin{array}{c}\text { Period } \\
\text { (days) } \\
(2)\end{array}$ & $\begin{array}{l}\text { GCVS Class } \\
\text { (3) }\end{array}$ & $\begin{array}{l}\text { Harris Class } \\
\text { (4) }\end{array}$ & $\begin{array}{l}n_{c} \\
(5)\end{array}$ & $\begin{array}{c}\sigma_{V}, \sigma_{R} \\
\text { (mmag) } \\
(6)\end{array}$ & $\begin{array}{l}n_{n} \\
(7)\end{array}$ & $\begin{array}{c}\text { HJD }-2,400,000 \\
(8)\end{array}$ & $\begin{array}{l}N \\
(9)\end{array}$ & $\begin{array}{l}\text { Source of Phot. } \\
\text { (10) }\end{array}$ \\
\hline V484 Mon............ & 3.13 & CEP & $\ldots$ & 4 & 9,16 & 2 & $51914-53082$ & $69(24)$ & $1,2,3$ \\
\hline DQ And................... & 3.20 & CWB: & II & 2 & 9,11 & 10 & $51800-52886$ & $95(44)$ & $3,4,5$ \\
\hline GL Cyg .................. & 3.37 & CEP & II & 6 & 12,17 & 4 & $52194-52906$ & $52(21)$ & 1,3 \\
\hline \multirow[t]{2}{*}{ FT Mon .................... } & 3.42 & DCEP & II & 4 & 7,7 & 7 & $48705-50376$ & $119(14)$ & 1,3 \\
\hline & & & & 2 & 7,7 & 3 & $51900-53101$ & $(40)$ & . \\
\hline EF Tau...................... & 3.45 & DCEP & $\ldots$ & 3 & 12,10 & 5 & $51900-52937$ & $138(38)$ & $1,3,5$ \\
\hline 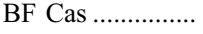 & 3.63 & DCEP & $\ldots$ & 5 & 6,7 & 7 & $52249-52905$ & $116(32)$ & $1,3,6$ \\
\hline \multirow[t]{2}{*}{ 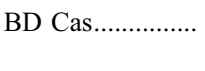 } & 3.65 & CWB & $\ldots$ & & $\mathrm{a}$ & & $46343-46762$ & 89 (14) & 4,6 \\
\hline & & & & 3 & 7,10 & 8 & $51800-52905$ & (37) & \\
\hline V572 Aq1 .............. & 3.77 & CWB & II & 4 & 11,10 & 3 & $52164-52866$ & $45(21)$ & 3,4 \\
\hline QY Cyg.................. & 3.89 & CWB: & II & 4 & 10,9 & $9^{b}$ & $51793-52886$ & $52(20)$ & 1,3 \\
\hline FM Del..................... & 3.95 & CWB & II & 4 & 11,9 & 5 & $51837-52859$ & $59(40)$ & 3 \\
\hline 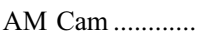 & 4.00 & CEP & $\ldots$ & 3 & 12,7 & $6^{\mathrm{b}}$ & $51900-53261$ & $152(57)$ & 1,3 \\
\hline V912 Aq1 ............... & 4.40 & DCEP & $\ldots$ & 2 & 22,30 & $4^{b}$ & $48573-52899$ & $45(22)$ & 3 \\
\hline V383 Cyg................ & 4.61 & CWB: & $\ldots$ & 2 & 17,12 & $6^{\mathrm{b}}$ & $51815-52906$ & $65(23)$ & 1,3 \\
\hline \multirow[t]{2}{*}{ 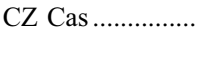 } & 5.66 & DCEP & $\ldots$ & 2 & 14,7 & 5 & $48654-50426$ & $88(14)$ & 1 \\
\hline & & & & 1 & 8,10 & 8 & $51921-52914$ & (31) & $\ldots$ \\
\hline V394 Cep............ & 5.69 & CWB: & $\ldots$ & 3 & 9,8 & 7 & $51837-52852$ & $41(41)$ & $\ldots$ \\
\hline 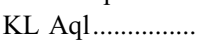 & 6.11 & DCEP & $\ldots$ & 2 & 15,22 & 3 & $52194-52886$ & $91(17)$ & $1,4,7,8$ \\
\hline TX Del' & 6.17 & CWB: & II & 1 & 12,18 & 3 & $52088-52906$ & $96(20)$ & $4,7,8,9,10$ \\
\hline V733 Aq1 ............ & 6.18 & DCEP & II & 3 & 6,7 & 4 & $52485-53074$ & $110(17)$ & 1,4 \\
\hline \multirow[t]{2}{*}{ 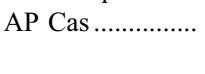 } & 6.85 & DCEP & $\ldots$ & 2 & 10,12 & 4 & $48605-50426$ & $60(12)$ & 1 \\
\hline & & & & 3 & 13,12 & 6 & $52309-52906$ & $(28)$ & $\ldots$ \\
\hline IT Cep ...................... & 7.35 & CWB: & $\ldots$ & 2 & 9,9 & 11 & $51793-52906$ & $43(43)$ & $\ldots$ \\
\hline BB Her..................... & 7.51 & DCEP & $\ldots$ & 2 & 16,11 & 4 & $52456-52844$ & $69(12)$ & $1,4,8$ \\
\hline \multirow[t]{2}{*}{ CD Cas.................... } & 7.80 & DCEP & $\ldots$ & 2 & 10,13 & 6 & $49634-51801$ & $59(11)$ & $\ldots$ \\
\hline & & & & 3 & 9,10 & 7 & $52194-52886$ & $(48)$ & $\ldots$ \\
\hline
\end{tabular}

${ }^{a}$ The data for BD Cas between JD 2,446,343 and 2,446,762 were obtained with the Behlen Observatory photoelectric photometer as described in Loomis et al. (1988). The comparison stars were not observed on any photometric nights. The zero points were set by fitting to the later Behlen Observatory data.

b The zero point of our $V$ magnitudes was adjusted to produce agreement with earlier photometry.

${ }^{c}$ Harris \& Welch (1989) showed that TX Del is a single-line spectroscopic binary. They note that the orbital parameters suggest that mass transfer has had a significant effect on the evolution of the star.

References.-(1) Berdnikov 1987, 1992a, 1992b, 1992c, 1992d, 1992e, 1992f, 1993a, 1993b; (2) Berdnikov \& Turner 1995; (3) Henden 1980, 1996a, 1996b; (4) Szabados 1977, 1980, 1991; (5) Schmidt et al. 1995; (6) Schmidt \& Reiswig 1993; (7) Pel 1976; (8) Harris 1980; (9) Moffett \& Barnes 1984; (10) Berdnikov \& Voziakova 1995.

indicates how many of them are from the present photometry. Finally, column (10) provides references to the sources of the earlier photometry.

In Table 2 we list the new observations. These data are without the zero-point adjustment mentioned above. The first author will provide complete tabulations of all of the data for each star on request.

\section{PROPERTIES OF THE LIGHT CURVES}

In Table 3 we list various parameters derived from the photometry. The first column lists the star names, while the second

TABLE 2

РнотомETRIC DAtA

\begin{tabular}{ccccc}
\hline \hline Star & HJD & $V$ & $R$ & $V-R$ \\
\hline V484 Mon........... & 51914.640 & 13.898 & 13.165 & 0.736 \\
& 51921.672 & 13.471 & 12.778 & 0.696 \\
& 51929.662 & 14.061 & 13.277 & 0.788 \\
& 51942.677 & 13.952 & 13.198 & 0.759 \\
& 51956.647 & 13.728 & 12.996 & 0.731 \\
\hline
\end{tabular}

Note.-Table 2 is published in its entirety in the electronic edition of the Astronomical Journal. A portion is shown here for guidance regarding its form and content. column gives the adopted periods, most of which are newly determined. The footnotes indicate what data were used in determining these periods. Column (3) gives upper limits on the uncertainty of the periods in units of the last cited digit of the period. In column (4) we list $\Delta P$, which is the difference between the period in column (2) and the period from the GCVS. It is also expressed in units of the last digit of the period. In column (5) we list the epoch of maximum based on the most recent photometry. Sampling and scatter can make the time of maximum uncertain. To address this in a consistent fashion, we have estimated the time of maximum by linearly extrapolating the rising and declining branches of the light curve to the point where they meet. There are stars with rather flat maxima or double maxima, which makes the selection of the time of maximum ambiguous. The reader can judge how this was handled by examining the light curves in Figure 1. In columns (6)-(9), we list the intensity mean magnitudes and the amplitudes in $V$ and $R$. Finally, we have provided the phase of minimum light in column (10). This was determined by linear extrapolation as for the maximum. Some of the stars have poorly defined minima, and again the reliability of the phases of minimum can be assessed by looking at the light curves themselves.

Figure 1 presents plots of the light curves, including both our data and those from the references in column (11) of Table 1. The 
TABLE 3

The Light-Curve Parameters

\begin{tabular}{|c|c|c|c|c|c|c|c|c|c|c|}
\hline $\begin{array}{l}\text { Star } \\
(1)\end{array}$ & $\begin{array}{c}\text { Period } \\
\text { (days) } \\
(2)\end{array}$ & $\begin{array}{l}\sigma_{P} \\
(3)\end{array}$ & $\begin{array}{l}\Delta P \\
(4)\end{array}$ & $\begin{array}{c}\mathrm{HJD}_{\max }-2,450,000 \\
(5)\end{array}$ & $\begin{array}{c}\langle V\rangle \\
(\mathrm{mag}) \\
(6)\end{array}$ & $\begin{array}{c}\langle R\rangle \\
(\mathrm{mag}) \\
(7)\end{array}$ & $\begin{array}{c}\Delta V \\
(\mathrm{mag}) \\
(8)\end{array}$ & $\begin{array}{c}\Delta R \\
(\mathrm{mag}) \\
(9)\end{array}$ & $\begin{array}{c}\phi_{\min } \\
(10)\end{array}$ & $\begin{array}{c}\text { Notes } \\
(11)\end{array}$ \\
\hline V484 Mon.......... & 3.13542 & 5 & 19 & 1915.12 & 13.73 & 13.02 & 0.84 & 0.66 & 0.74 & a \\
\hline DQ And.............. & 3.20063 & 2 & 7 & 2662.55 & 11.68 & 11.29 & 0.84 & 0.64 & 0.82 & a \\
\hline GL Cyg .............. & 3.37080 & 5 & 11 & 2781.75 & 13.73 & 12.95 & 0.75 & 0.57 & 0.74 & a \\
\hline FT Mon ............... & 3.42180 & 5 & $\mathrm{~b}$ & 2639.76 & 12.73 & 12.10 & 1.17 & 0.90 & 0.81 & a \\
\hline EF Tau................. & 3.44825 & 10 & 10 & 2648.64 & 13.09 & 12.50 & 0.72 & 0.56 & 0.83 & a \\
\hline BF Cas .................. & 3.63046 & 3 & 17 & 2569.79 & 12.47 & 11.70 & 0.89 & 0.70 & 0.82 & $\mathrm{a}$ \\
\hline BD Cas............... & 3.6508 & 1 & -1 & 2858.77 & 11.06 & 10.24 & 0.36 & 0.27 & 0.51 & $\mathrm{c}$ \\
\hline V572 Aql ........... & 3.76784 & 3 & 14 & 2852.49 & 11.12 & 10.68 & 0.35 & 0.29 & 0.61 & d \\
\hline QY Cyg.............. & 3.89188 & 5 & 0 & 2618.56 & 14.64 & 14.04 & 0.98 & 0.82 & 0.79 & e \\
\hline FM Del............... & 3.95452 & 100 & 0 & 2781.85 & 12.44 & 12.06 & 0.71 & 0.40 & 0.62 & $\mathrm{f}$ \\
\hline AM Cam ............ & 3.99715 & 3 & -5 & 1921.51 & 13.52 & 12.57 & 0.60 & 0.49 & 0.80 & a \\
\hline V912 Aql .......... & 4.40034 & 10 & 0 & 2864.59 & 11.38 & 10.01 & 0.85 & 0.67 & 0.79 & e \\
\hline V383 Cyg.......... & 4.61230 & 5 & 9 & 2822.87 & 10.90 & 9.95 & 0.59 & 0.46 & 0.76 & a \\
\hline CZ Cas .............. & 5.6646 & 1 & 2 & 2618.80 & 11.74 & 10.89 & 0.81 & 0.67 & 0.76 & a \\
\hline V394 Cep............ & 5.688 & 2 & $\mathrm{~g}$ & 2810.49 & 13.93 & 13.00 & 0.84 & 0.64 & 0.76 & a \\
\hline KL Aql............... & 6.108015 & 100 & 0 & 2500.51 & 10.18 & 9.65 & 0.75 & 0.59 & 0.74 & $\mathrm{e}$ \\
\hline TX Del ................ & 6.165907 & 5000 & 0 & 2781.44 & 9.03 & 8.58 & 0.80 & 0.60 & 0.70 & $\mathrm{f}$ \\
\hline V733 Aql .......... & 6.178748 & 200 & 0 & 2822.85 & 9.96 & 9.46 & 0.51 & 0.40 & 0.72 & e \\
\hline AP Cas ................. & 6.8468 & 2 & -2 & 2662.84 & 11.57 & 10.76 & 0.63 & 0.48 & 0.72 & a \\
\hline IT Cep............... & 7.34744 & 200 & 0 & 2832.66 & 13.57 & 12.59 & 0.57 & 0.45 & 0.74 & e \\
\hline BB Her............... & 7.507945 & 200 & 0 & 2769.87 & 10.08 & 9.44 & 0.66 & $\ldots$ & 0.69 & e \\
\hline CD Cas................ & 7.802 & 1 & 1 & 2781.71 & 10.76 & 9.87 & 0.84 & 0.65 & 0.73 & a \\
\hline
\end{tabular}

Note.-Table 3 is also available in machine-readable form in the electronic edition of the Astronomical Journal.

a The new period and light-curve parameters were determined from all of the available data.

b The GCVS period of 4.834225 days is evidently in error.

c All the data were used to determine the period for BD Cas, but only the present data and that from Schmidt \& Reiswig (1993) were used to determine the other light-curve parameters.

d For V572 Aql, the data of Szabados (1977; JD 2,441,568 to 2,441,944) are systematically 0.09 mag fainter than ours, while those of Henden (1980; JD 2,443,630

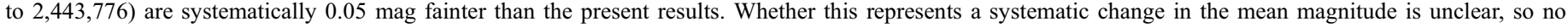

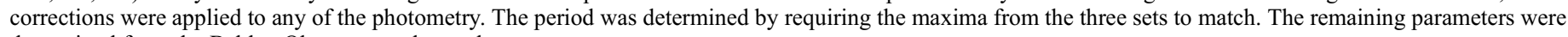
determined from the Behlen Observatory data only.

e The new period was not significantly different from the GCVS period, so the latter was used. The light-curve parameters were determined from all the data.

${ }^{\mathrm{f}}$ For TX Del, the new period was not significantly different from the GCVS period, so the latter was adopted. The light-curve parameters were determined from the present data only.

$\mathrm{g}$ The GCVS does not list a period for V394 Cep.

ephemerides in Table 3 were used for phasing the data. For some stars, different symbols distinguish particular subsets of the data as discussed below in $\S$ 4.2.1.

We have fitted Fourier series to all our light curves. The order of the adopted fit for each star was selected to avoid overfitting of the data while minimizing the scatter. The Fourier parameters (see Simon \& Lee 1981 for definitions) are listed in Table 4. The errors were calculated using the scheme of Petersen (1986). Larger errors are generally associated with light curves with greater scatter.

To measure the scatter in the light curves, we have calculated the standard deviations of individual data points about the fitted curve. When the light curve is very asymmetric, the Fourier fit is generally poor from before minimum until after maximum light. In those cases, we have removed points where the fit was poor and calculated the scatter with the remainder of the data set. The standard deviations are listed in the last column of Table 4.

\section{DISCUSSION}

\subsection{The Form of the Light Curves}

In Papers I and II, morphological classification of the light curves proved to be useful in understanding the various types of stars present in the sample. For the short-period stars discussed in Paper II, we adopted the classification scheme of Diethelm $(1983,1990)$. The significance of his classes was demonstrated by the fact that they separated type I from type II stars and, among the latter, were well correlated with metallicity. Similarly, in Paper I we placed the light curves of long-period Cepheids into several groups that were useful in distinguishing type I from type II Cepheids. Our class-D light curves for the long-period stars and Diethelm's AHB1 and $\mathrm{C} \delta$ light curves are quite similar. The majority of the light curves in Figure 1 fit into these categories. Exceptions are V572 Aql, BD Cas, CD Cas, TX Del, FM Del, and BB Her. V572 Aql, FM Del, and TX Del resemble our class A, stars with a broad flat maximum and a symmetric minimum. BD Cas, a low- $Z$ star, might also be placed in our class A on the basis of the symmetry of its light curve around minimum light. CD Cas and BB Her differ from our type-D stars in that they have a bump on declining light. This is normal for classical Cepheids with periods near 8 days.

In Figure 2 we have plotted three of the Fourier parameters from Table 4 against period. Additional stars from Moffett \& Barnes (1985), Antonello et al. (1990), and Mantegazza \& Poretti (1992) have been included for comparison. We have also plotted mean sequences for classical Cepheids measured from Figures 3, 4, and 5 of Mantegazza \& Poretti (1992). These authors have identified distinct sequences with fundamental and first overtone (referred to as s-Cepheids) pulsators. This identification has been confirmed by Fourier decomposition of multimode Cepheids (Poretti \& Pardo 1997) and by reference to the 

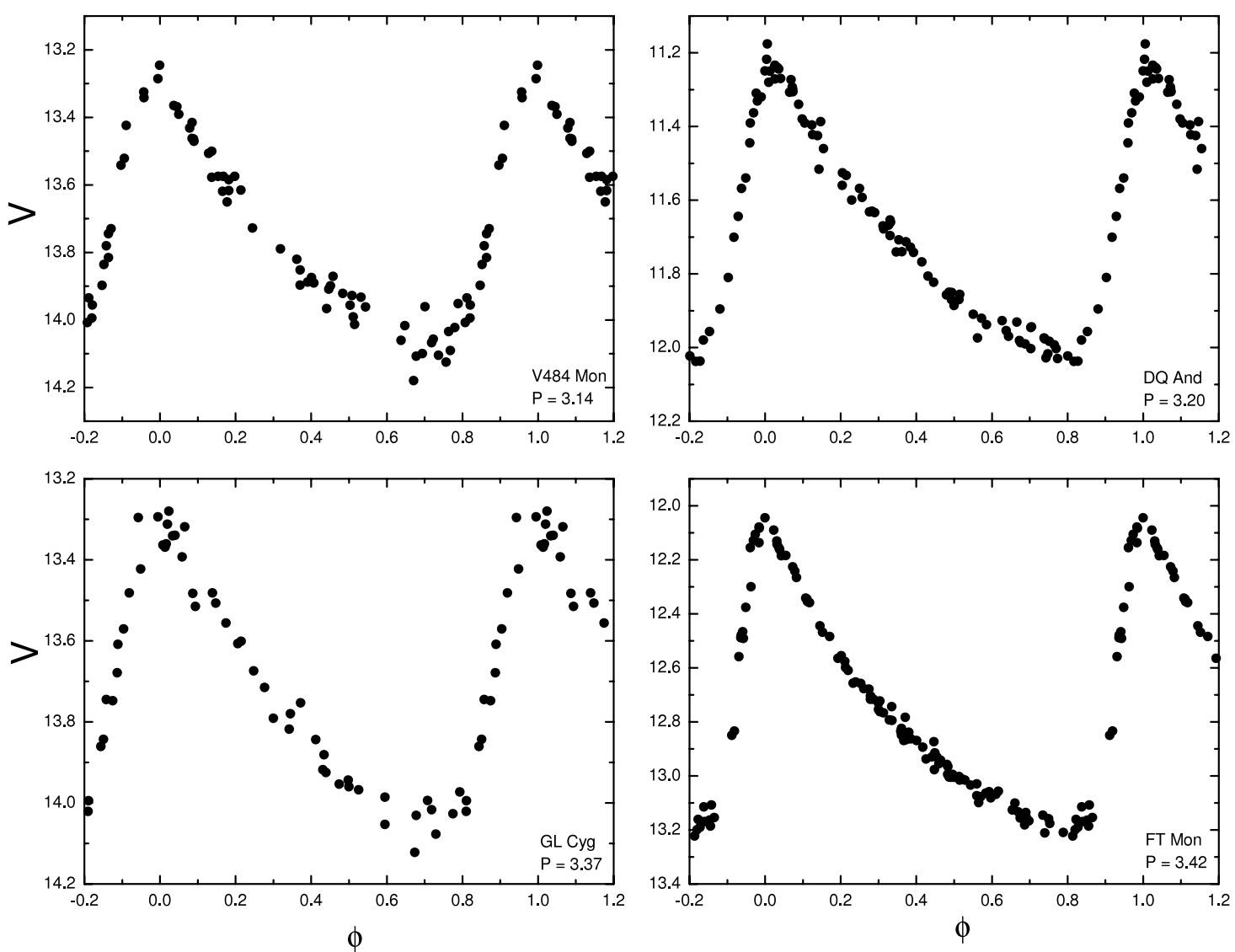

FIG. $1 a$
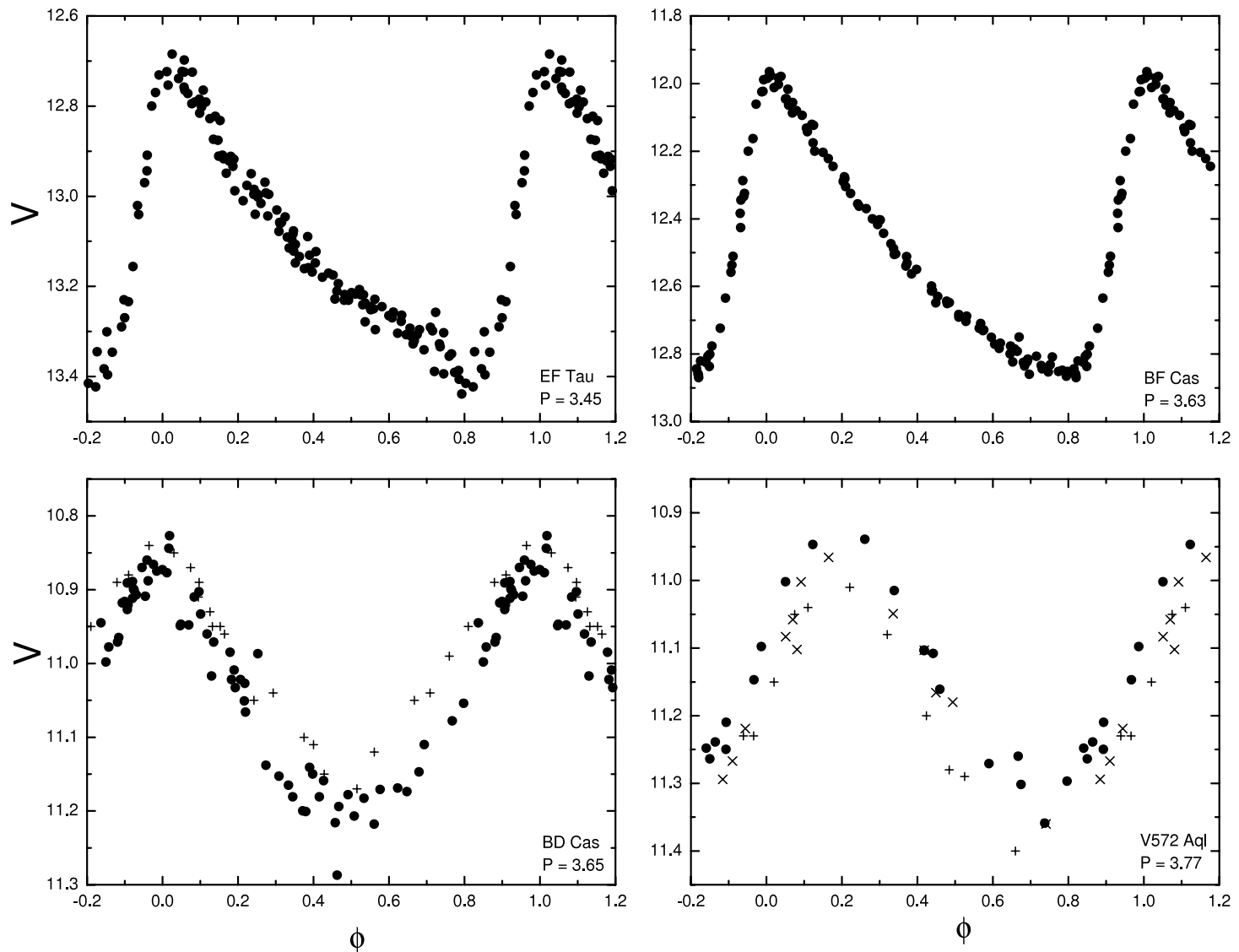

FIG. $1 b$

Fig. 1. - V-magnitude light curves for the stars listed in Table 1. In some cases different symbols denote different subsets of data as described in the text. 

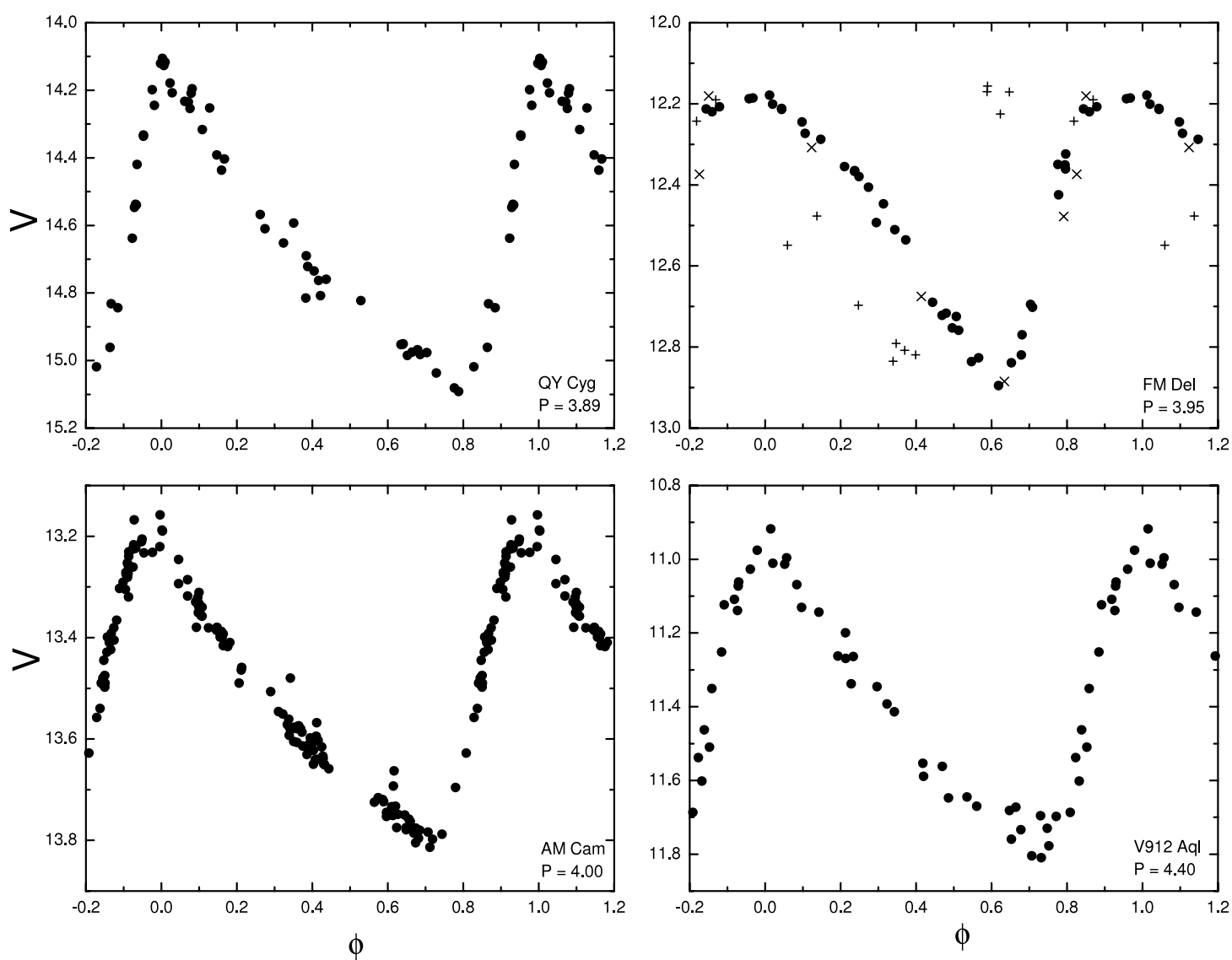

FIG. $1 c$
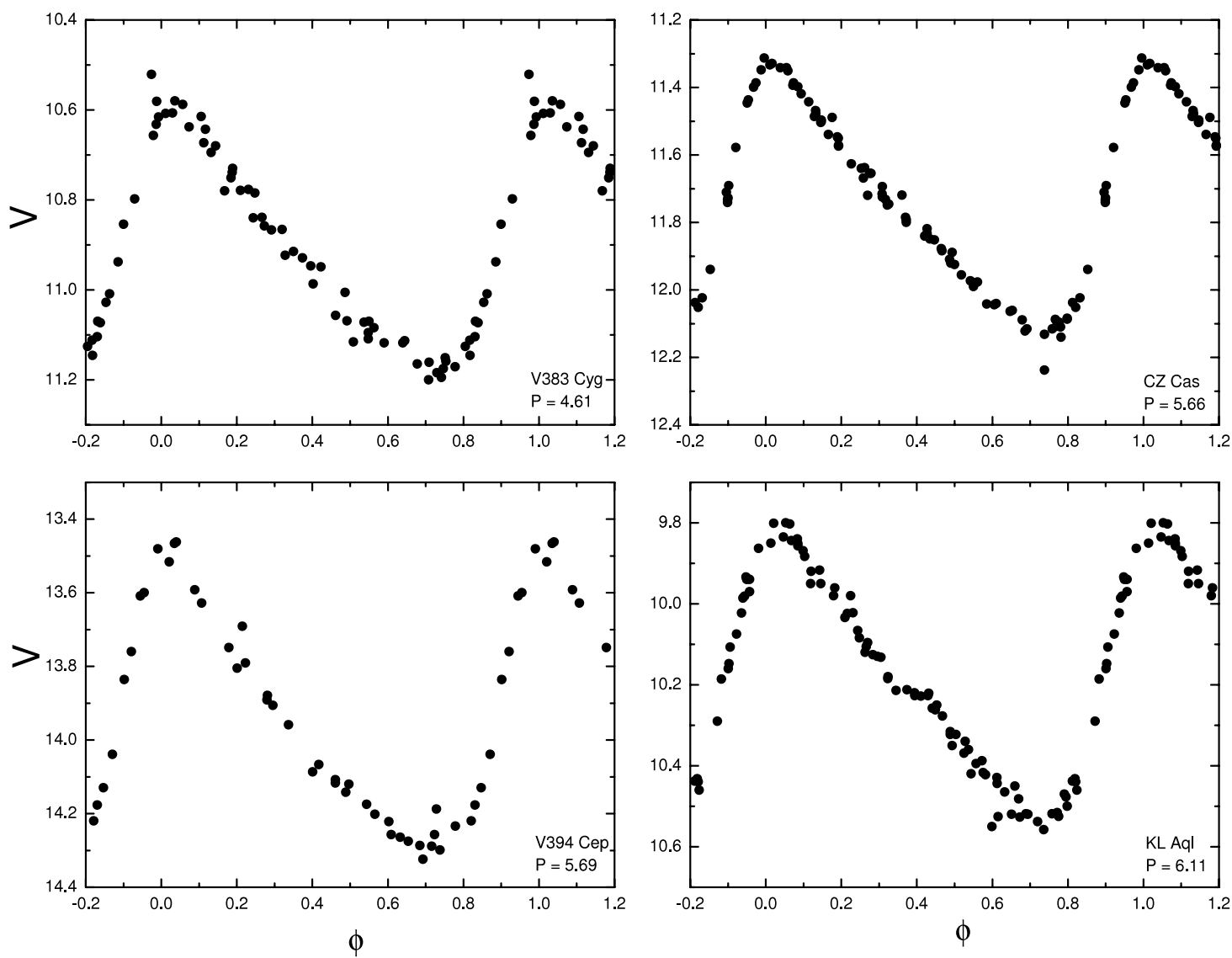

FIG. $1 d$ 

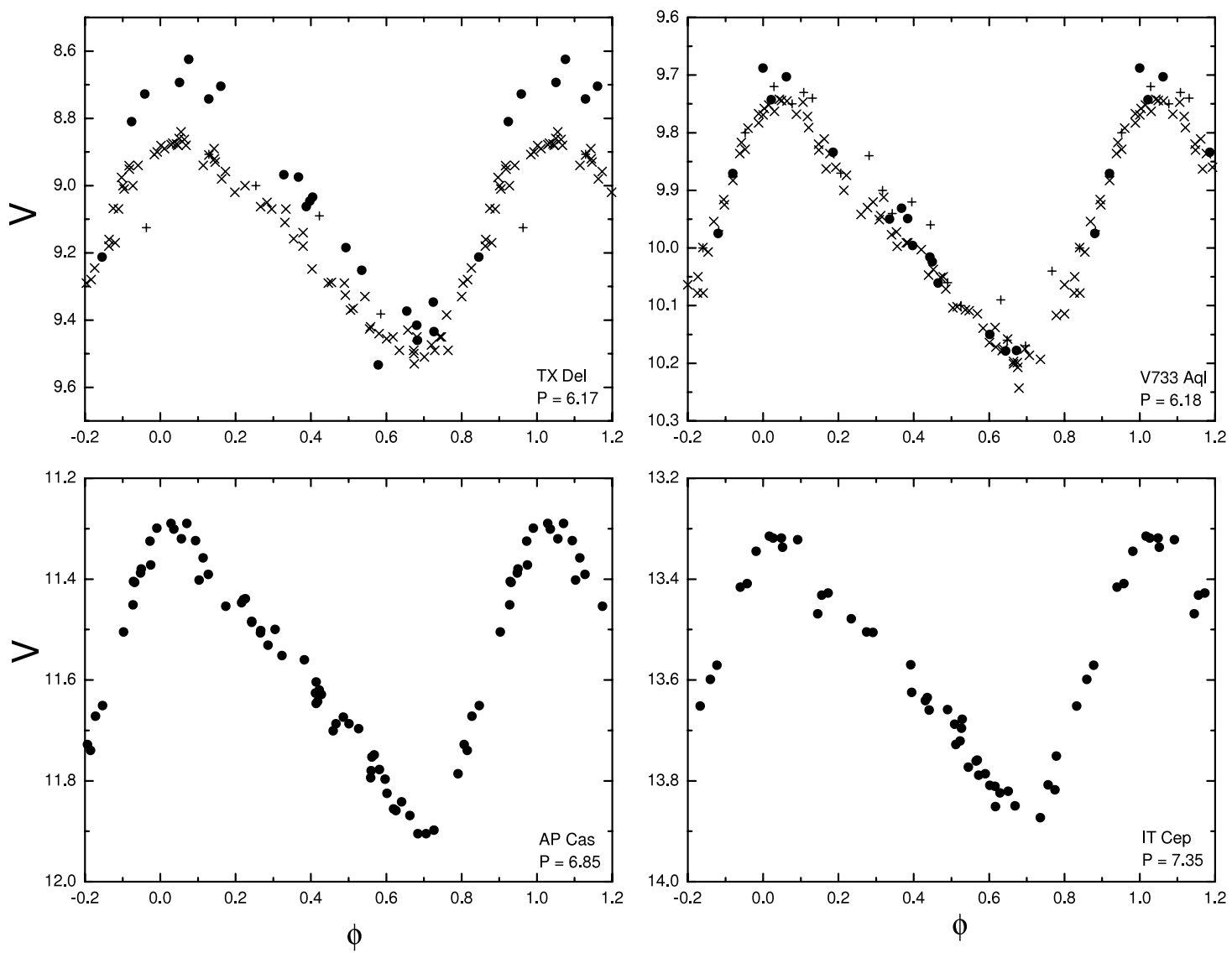

FIG. $1 e$
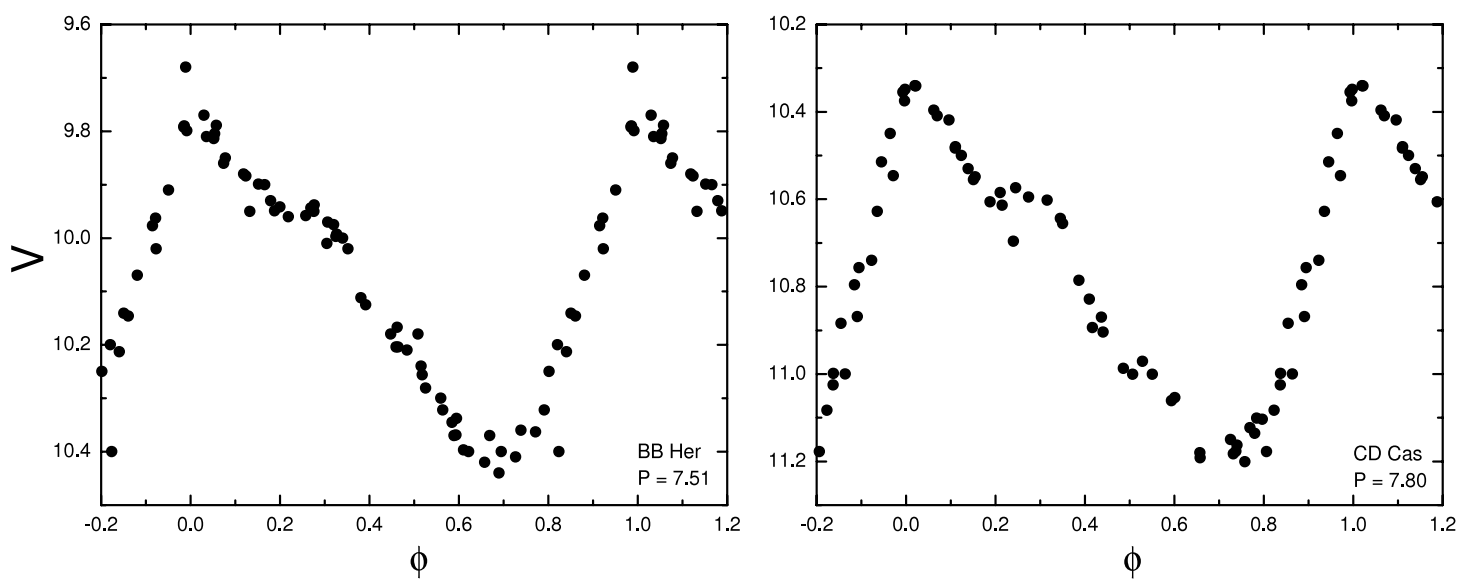

FIG. $1 f$

( $\log P-V$ ) diagram for SMC and LMC Cepheids (Alcock et al. 1999; Udalski et al. 1999a, 1999b). Mantegazza \& Poretti only identified stars with periods less than 5 days as overtone pulsators. However, there are stars at longer period that fall below the locus of the majority of the stars and define an obvious extension of the overtone stars. Hence, we have extended the overtone sequences to the point where they intersect with the fundamental sequences.

An examination of Figure 2 shows that of our low- $Z$ stars, only one, BD Cas, is clearly an overtone pulsator. All three of the diagrams are consistent in indicating the mode of the low- $Z$ stars.

Smith et al. (1992) showed that, among SMC Cepheids, overtone pulsators have significantly smaller amplitudes than fundamental pulsators. To explore this we have plotted the $V$ amplitudes from column (8) of Table 3 against the periods in Figure $3 a$. The present sample has been supplemented by the amplitudes of other stars in the same period range from the DDO Database of Galactic Classical Cepheids. ${ }^{1}$ The frequency distribution of the amplitudes is plotted in Figure $3 b$. Although a wide range is present, the distribution has a clear minimum at about $\Delta V=0.4-0.45$. Assuming that it separates the fundamental from the overtone pulsators, we again find that of our low- $Z$ stars, only BD Cas is an overtone pulsator.

It is not clear how well the high- $Z$ stars should be expected to follow the classical Cepheid sequences in the Fourier diagrams.

\footnotetext{
${ }^{1}$ Available at http://www.astro.utoronto.ca/DDO/research/cepheids.
} 
TABLE 4

The Fourier Parameters

\begin{tabular}{|c|c|c|c|c|c|c|c|c|c|c|c|c|c|}
\hline $\begin{array}{l}\text { Star } \\
(1)\end{array}$ & $\begin{array}{l}R_{21} \\
(2)\end{array}$ & $\begin{array}{c}\sigma \\
(3)\end{array}$ & $\begin{array}{l}R_{31} \\
(4)\end{array}$ & $\begin{array}{c}\sigma \\
(5)\end{array}$ & $\begin{array}{c}R_{41} \\
(6)\end{array}$ & $\begin{array}{c}\sigma \\
(7)\end{array}$ & $\begin{array}{l}\phi_{21} \\
(8)\end{array}$ & $\begin{array}{c}\sigma \\
(9)\end{array}$ & $\begin{array}{c}\phi_{31} \\
(10)\end{array}$ & $\begin{array}{c}\sigma \\
(11)\end{array}$ & $\begin{array}{c}\phi_{41} \\
(12)\end{array}$ & $\begin{array}{c}\sigma \\
(13)\end{array}$ & $\begin{array}{c}\sigma_{V} \\
(14)\end{array}$ \\
\hline V484 Mon.......... & 0.42 & 0.02 & 0.17 & 0.02 & 0.10 & 0.02 & 4.19 & 0.07 & 2.20 & 0.13 & 6.08 & 0.23 & 0.040 \\
\hline DQ And............... & 0.42 & 0.01 & 0.24 & 0.01 & 0.11 & 0.01 & 4.22 & 0.03 & 2.24 & 0.05 & 0.30 & 0.10 & 0.022 \\
\hline GL Cyg .............. & 0.35 & 0.03 & 0.16 & 0.03 & 0.09 & 0.02 & 4.22 & 0.09 & 2.14 & 0.17 & 0.31 & 0.29 & 0.040 \\
\hline FT Mon ............... & 0.48 & 0.01 & 0.28 & 0.01 & 0.16 & 0.01 & 4.16 & 0.03 & 2.10 & 0.04 & 0.04 & 0.06 & 0.028 \\
\hline EF Tau................. & 0.48 & 0.02 & 0.27 & 0.01 & 0.13 & 0.01 & 4.22 & 0.04 & 2.40 & 0.07 & 0.44 & 0.13 & 0.030 \\
\hline BF Cas .................. & 0.41 & 0.01 & 0.21 & 0.01 & 0.12 & 0.01 & 4.26 & 0.02 & 2.37 & 0.04 & 0.33 & 0.06 & 0.019 \\
\hline BD Cas................. & 0.16 & 0.03 & $\ldots$ & $\ldots$ & $\ldots$ & $\ldots$ & 2.95 & 0.22 & $\ldots$ & $\ldots$ & $\ldots$ & $\ldots$ & 0.026 \\
\hline V572 Aql ........... & 0.09 & 0.03 & $\ldots$ & $\ldots$ & $\ldots$ & $\ldots$ & 3.93 & 0.36 & $\ldots$ & $\ldots$ & $\ldots$ & $\ldots$ & 0.021 \\
\hline QY Cyg.............. & 0.49 & 0.03 & 0.21 & 0.02 & 0.14 & 0.02 & 4.36 & 0.07 & 2.40 & 0.13 & 0.64 & 0.19 & 0.038 \\
\hline FM Del................. & 0.31 & 0.02 & 0.11 & 0.02 & 0.07 & 0.02 & 5.28 & 0.06 & 4.11 & 0.14 & 3.08 & 0.24 & 0.022 \\
\hline AM Cam ............. & 0.40 & 0.01 & 0.18 & 0.01 & 0.05 & 0.01 & 4.32 & 0.03 & 2.48 & 0.06 & 0.68 & 0.16 & 0.019 \\
\hline V912 Aql ........... & 0.34 & 0.03 & 0.20 & 0.03 & 0.04 & 0.03 & 4.32 & 0.10 & 2.49 & 0.16 & 1.11 & 0.80 & 0.046 \\
\hline V383 Cyg........... & 0.34 & 0.02 & 0.14 & 0.02 & 0.03 & 0.02 & 4.32 & 0.06 & 2.39 & 0.12 & 0.94 & 0.58 & 0.024 \\
\hline CZ Cas ................ & 0.38 & 0.01 & 0.15 & 0.01 & 0.07 & 0.01 & 4.48 & 0.03 & 2.48 & 0.06 & 0.71 & 0.12 & 0.018 \\
\hline V394 Cep........... & 0.36 & 0.02 & 0.15 & 0.02 & 0.10 & 0.02 & 4.22 & 0.06 & 2.38 & 0.12 & 6.06 & 0.18 & 0.026 \\
\hline KL Aql................ & 0.37 & 0.01 & 0.09 & 0.01 & 0.06 & 0.01 & 4.56 & 0.04 & 2.57 & 0.14 & 0.92 & 0.20 & 0.024 \\
\hline TX Del ................ & 0.27 & 0.04 & 0.09 & 0.04 & $\ldots$ & $\ldots$ & 4.79 & 0.17 & 3.18 & 0.44 & $\ldots$ & $\ldots$ & 0.044 \\
\hline V733 Aql ........... & 0.29 & 0.02 & 0.06 & 0.02 & 0.03 & 0.02 & 4.66 & 0.07 & 1.70 & 0.31 & 4.24 & 0.69 & 0.027 \\
\hline AP Cas ................... & 0.34 & 0.02 & 0.07 & 0.02 & 0.04 & 0.02 & 4.88 & 0.05 & 3.26 & 0.21 & 5.02 & 0.43 & 0.021 \\
\hline IT Cep .................. & 0.33 & 0.02 & 0.06 & 0.02 & 0.05 & 0.02 & 4.91 & 0.08 & 2.87 & 0.34 & 4.99 & 0.46 & 0.023 \\
\hline BB Her................ & 0.27 & 0.02 & 0.09 & 0.02 & 0.07 & 0.02 & 5.13 & 0.06 & 2.93 & 0.17 & 5.82 & 0.21 & 0.023 \\
\hline CD Cas................ & 0.27 & 0.02 & 0.20 & 0.02 & 0.09 & 0.02 & 4.88 & 0.08 & 2.90 & 0.11 & 5.75 & 0.21 & 0.035 \\
\hline
\end{tabular}
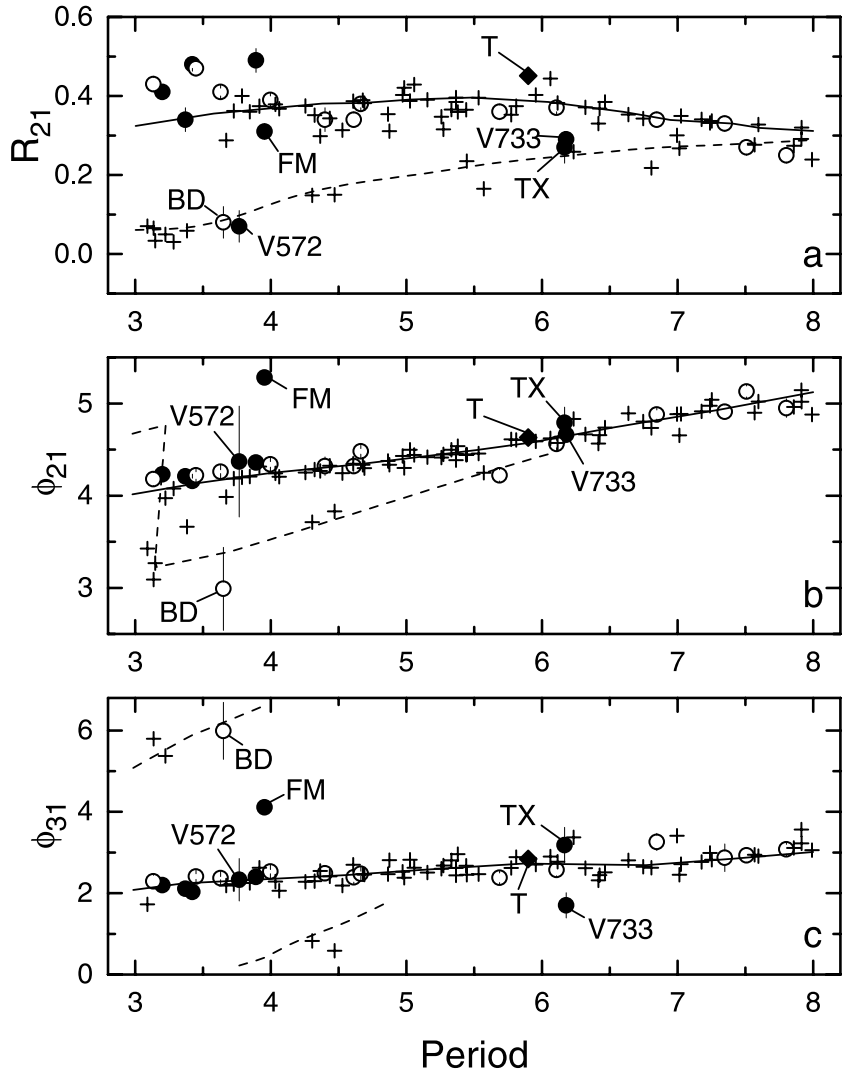

FIG. 2.-Fourier parameters plotted against period. Open and filled circles represent low- and high- $Z$ stars from the present sample, respectively. Error bars are shown when they are larger than the plotted symbol. Plus signs and a filled diamond represent low- and high- $Z$ stars from the literature, respectively. Mean curves are plotted for fundamental pulsators as solid lines and for overtone pulsators as dashed lines. Stars discussed in the text are labeled as follows: BD, BD Cas; FM, FM Del; TX, TX Del; V572, V572 Aql; V733, V733 Aql; and T, T Ant.
Among the six short-period high- $Z$ stars, four agree reasonably well with the fundamental curves in all three diagrams. All four also have large amplitudes, so we conclude they are likely to be fundamental pulsators.

V572 Aql falls near the overtone curve in the $R_{21}$ diagram and its amplitude is consistent with the overtone mode. On the other hand, it is on the fundamental curve in the phase diagrams. We note that the classical Cepheid overtone sequence in the $\phi_{21}$ diagram has a discontinuity near 3.1 days, which is likely to be associated with a mode resonance. It can be seen in Figure 3 of Mantegazza \& Poretti (1992) that there are several overtone pulsators scattered between the two branches of the sequences. If the resonance occurred at a period that was about a half-day longer for high- $Z$ stars, V572 Aql could be in this transition region. In the $\phi_{31}$ diagram, the overtone and the fundamental sequences for classical Cepheids intersect at a period of about 2.2 days (see Fig. 4 of Mantegazza \& Poretti). Thus, the overtone sequence would have to be shifted about 1.5 days to bring V572 Aql into agreement. These shifts are consistent with shifts suggested in Paper II for short-period, high- $Z$ stars. Further type II Cepheids in this period range, if they can be found, will need to be observed to clarify this issue. In the meantime, it seems likely that V572 Aql is an overtone pulsator on the basis of its amplitude.

FM Del is a likely fundamental mode pulsator on the basis of its amplitude and position in Figure $2 a$. However, in the phase diagrams, Figures $2 b$ and $2 c$, it is significantly removed from both the fundamental and the overtone curves. Given the size of our sample, we cannot determine whether this star is peculiar in some way or simply indicates that the curves for type II Cepheids differ significantly from those of the low- $Z$ stars even with the suggested period shifts.

The three longer period high- $Z$ stars also indicate that the sequences will need to be modified to accommodate the type II Cepheids. Only T Ant is consistently on the fundamental sequences in the Fourier diagrams and has a large amplitude. 

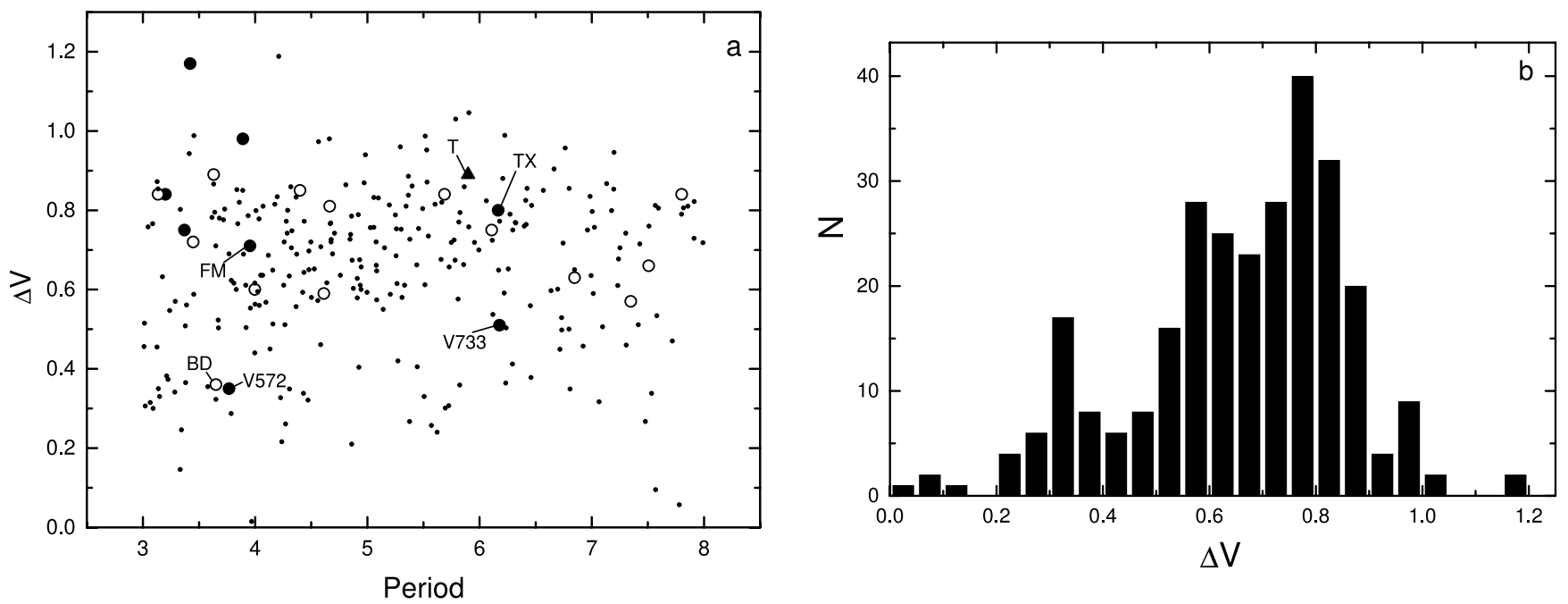

FIG. 3.- (a) $V$ amplitude plotted against period. Large symbols represent stars from the present sample and have the same meaning as in Fig. 2. Dots are amplitudes of stars from the DDO Database of Galactic Classical Cepheids. (b) Frequency distribution of the amplitudes plotted in $(a)$.

TX Del has a large amplitude and falls near the fundamental sequence in $\phi_{31}$, yet it is close to the overtone sequence in $R_{21}$. On the other hand, V733 Aql lies on the overtone sequence in $R_{21}$ and in $\phi_{31}$ if we shift the curve as suggested above. Its amplitude is only marginally in the range of the fundamental pulsators so it seems likely to be an overtone pulsator. However, the pulsation mode of both of these stars must remain uncertain at present.

We conclude that Fourier decomposition, in conjunction with amplitude, does serve to identify some unusual intermediateperiod Cepheids. However, it does not seem to distinguish between type I and type II Cepheids in most cases. Similarly, the appearance of the light curves can be used to distinguish a few type II stars but is again of limited usefulness. These conclusions are compromised to some extent by the possibility that some low- $Z$ Cepheids may be of type II and some high- $Z$ stars may be classical Cepheids (see Schmidt et al. 2003a).

\subsection{Stability of the Pulsation}

\subsubsection{Changes in Light-Curve Shape}

An examination of Figure 1 reveals excess scatter in a number of the light curves. Generally this is due to differences among several of the data sets plotted rather than scatter within a particular data set. Accordingly, we have distinguished various data sets that contribute unduly to the scatter by different symbols. We discuss these cases individually here.

$B D$ Cas.-For BD Cas (Fig. 1b), we have plotted data from Szabados (1977; between JD 2,441,566 and 2,442,300) as crosses and the data from Schmidt \& Reiswig (1993; JD 2,447,041 to $2,447,926$ ) and the present photometry (JD 2,446,343 to $2,446,762$ and $2,451,800$ to $2,452,905$ ) as circles. Clearly, the light-curve shape and the amplitude differ between the earlier and the later data. This suggests long-term changes in the light curve of this star.

V572 Aql.-For V572 Aql (Fig. 1b), the data of Szabados (1977; JD 2,441,567 to JD 2,442,225) are plotted as plus signs, that of Henden (1980; JD 2,443,630 to 2,443,810) as crosses, and the present photometry (from JD 2,452,164 to 2,452,866) as circles. It can be seen that there is a progression toward brighter mean magnitudes with time; the Szabados and Henden magnitudes average 0.09 and 0.045 mag fainter than the present photometry, respectively. This could represent a genuine brightening of the star or could be due to zero-point errors. We have not chosen to correct any of the data sets, since it is not clear which of these alternatives is more likely. Whatever the cause of this change, there is no appreciable change in the light-curve shape.

FM Del.-For FM Del (Fig. 1c), the portion of Henden's (1996b) data from JD 2,446,650 to $2,447,457$ are denoted by plus signs and the portion from JD $2,449,605$ to $2,450,286$ by crosses, while the present data (from JD 2,451,837 to 2,452,859) are represented by circles. It can be seen that Henden's earlier photometry appears shifted in phase relative to the present data. However, there is no indication of a change in light-curve shape.

TX Del.-For TX Del, five different sets of data taken between JD 2,440,753 and JD 2,444,942 (Pel 1976; Harris 1980; Szabados 1980; Moffett \& Barnes 1984; Szabados 1991; represented by crosses in Fig. 1e) produce a reasonably tight light curve with a single period. However, the five points from Berdnikov \& Voziakova (1995; represented by plus signs) that were taken between JD $2,449,624$ and $2,449,634$ require a period change to fit that curve. The data from the present study taken seven to nine years later (circles) do not indicate a period change in as much as the maximum and minimum match the older data. However, our data are brighter than the older data. We have not attempted to adjust our zero point, since the difference required would be implausibly large. In addition, the amplitude of our data is clearly larger than indicated by the earlier photometry.

V733 Aql.-For V733 Aql (Fig. 1e), the data of Szabados (1980; JD 2,442,276 to 2,443,404) are represented by plus signs, the data of Berdnikov (1992a, 1992f, 1993a) and Berdnikov \& Voziakova (1995) by crosses (JD 2,446,617 to 2,449,636), and the present data (JD 2,452,485 to $2,453,674$ ) by circles. With the adopted period, the Szabados points on rising light match the later photometry but those during declining light and around minimum do not. In addition, there are discrepancies among the three sets of data around maximum light. This star may exhibit some changes in its light curve over long intervals of time.

Conclusions. - Of the five stars discussed here, only three, V733 Aql, BD Cas, and TX Del, seem to show a change in the light-curve shape over a long interval. BD Cas and TX Del are classed as type II Cepheids in the GCVS, while V733 Aql and TX Del are high- $Z$ stars. These stars as well as V572 Aql, which may be systematically brightening, should be observed in the future to clarify their long-term behavior. 


\subsubsection{Period Stability}

As in Papers I and II, we use the quantity $\Delta P$ as a measure of long-term period stability. In Paper II we assessed its reliability by referring to the period studies of Szabados $(1977,1991)$; we concluded that $\Delta P$ was a good measure of period stability. The present sample includes seven stars for which Szabados (1977, 1980, 1991) conducted period studies. The latest period from Szabados's study for each of these stars was adopted for the GCVS. Thus, any period changes we detect will have occurred since the epoch of his data.

For one star in common, TX Del, the error in our period was too large to determine a meaningful value of $\Delta P$. For three of the stars, Szabados found constant periods. Of these we found $\Delta P=0$ for two, KL Aql and V733 Aql, and a small change, $\Delta P=0.00007 \pm 0.00002$ days, for the third, DQ And. For each of the other three stars Szabodos found two intervals of constant period separated by an abrupt period change. In two of these, BD Cas and BB Her, we found periods essentially in agreement with the GCVS (or the Szabados period after the period change), indicating that no further period changes have occurred. For the third, V572 Aql, we found an increase in the period of $0.00014 \pm$ 0.00003 days, while Szabados reported a decrease of -0.000903 days. Some Cepheids have been observed to show both increases and decreases in period over long intervals, so this result is not surprising. However, it is clear from this comparison that our determination of $\Delta P$ is subject to vagaries due to the rather long intervals between period changes.

In Figure $4 a$ we have plotted $\Delta P$ against period for most of the stars in our sample. Those with period uncertainties larger than 0.0002 days were not plotted because the resulting values of $\Delta P$ are not meaningful for our discussion. It is noteworthy that the period changes are all less that 0.0002 days in absolute value. We also note that the majority of the period changes are positive. For this reason, we only consider the absolute magnitudes in what follows.

This diagram is best understood by comparison with the corresponding plots in Papers I and II. Of the 24 short-period stars discussed in Paper II, four had period changes much larger than any in the present sample, while the rest are comparable to those found here. On the other hand, the stars in the long-period range (Paper I) exhibit period changes considerably larger than those from the present study; the smallest value of $|\Delta P|$ among the long-period stars is more than twice the largest from the present sample. In fact, there is a rather sudden change in the period stability between the longest period represented here, 7.8 days, and the shortest period of the sample in Paper I, 8.8 days. This is true for both the low- $Z$ and high- $Z$ stars.

In the context of distinguishing between type I and type II Cepheids, it is noteworthy that, unlike the short-period and the long-period samples, no stars stand out as having particularly large values of $|\Delta P|$. Furthermore, there is no apparent separation between the high- and the low- $Z$ stars. While this could be explained by the particular sample represented (e.g., all of the stars could be type II Cepheids, of which a few are close to the Galactic plane), it seems more likely that period stability is not a good discriminator of type in this period range.

\subsubsection{Light-Curve Scatter}

In Figure $4 b$ we have plotted the scatter in the light curve, $\sigma_{V}$, against period. For comparison with the longer and shorter period stars, we note that in Papers I and II we regarded stars with $\sigma_{V} \leq 0.04-0.045$ as exhibiting excess scatter. By this standard, none of the stars shown in Figure 4 stand out. We should
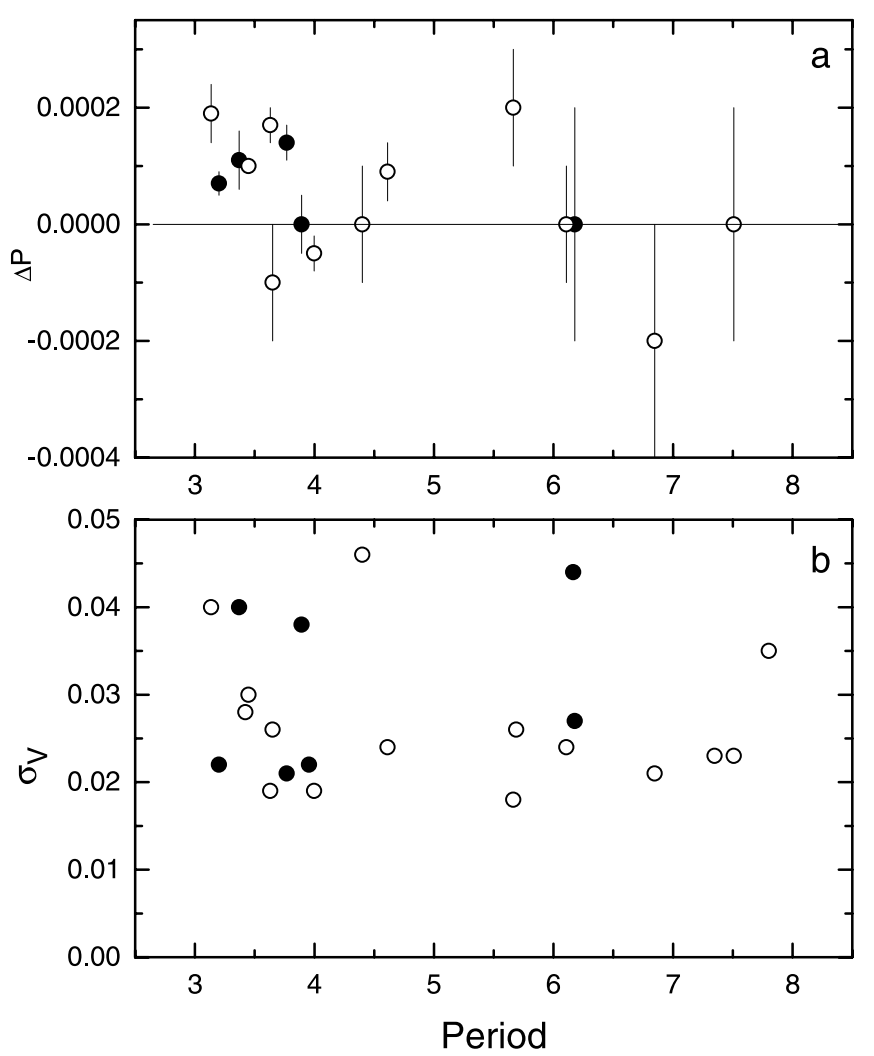

FIG. 4.- Period stability parameters plotted against period. The symbols have the same meaning as in Fig. 2.

note that in measuring the scatter of several stars, we have only used the present photometry. For this reason, our values $\sigma_{V}$ for stars like V912 Aql, V572 Aql, and TX Del are relatively small in spite of large changes over a number of decades. We conclude that light-curve scatter is not useful for distinguishing type I from type II Cepheids in the intermediate-period range.

\section{CONCLUSIONS}

In the previous sections, five stars were mentioned as unusual with regard to one or more of the properties discussed: V572 Aql (light curve, location in Fourier and amplitude diagrams, possible long-term behavior), FM Del (light curve, location in Fourier and amplitude diagrams), TX Del (light curve, location in Fourier and amplitude diagrams, long-term behavior), BD Cas (light curve, long-term behavior), and V733 Aql (location in Fourier and amplitude diagrams, long-term behavior). In addition, Schmidt et al. (2003b) found that the $\mathrm{H} \alpha$ absorption line in FM And was abnormally weak and the differential velocity was high compared with other stars of similar period. They concluded that it was a type II Cepheid on this basis. All of these stars are high- $Z$ stars except BD Cas and all are classified in the GCVS as type II Cepheids except V733 Aql.

Assuming that the five stars listed in the previous paragraph are all type II Cepheids, our discussion indicates that the shortperiod type II Cepheids or BL Her stars extend to periods of about 4 days and that long-period type II Cepheids or W Vir stars with periods as short as 6.2 days exist. Although we cannot argue that there are no type II Cepheids between these periods, it is worth noting that Harris (1985) lists only eight high- $Z$ stars in this interval. These stars should be investigated to establish their status. In the meantime, we suggest that the period division between these two groups should be put in this range. 
We made extensive use of the McMaster Cepheid Photometry and Radial Velocity Data Archive in both the selection of stars for the program and in locating photometric data for use in this paper. We are appreciative of the efforts on the part of Douglas Welch in providing this resource. This material is based on work supported by the National Science Foundation under grant 0097353. We are grateful to Roger Kirby, the Chair of the Department of Physics and Astronomy at the University of Nebraska, for his long and continued support of the operation of Behlen Observatory.
Alcock, C., et al. 1999, ApJ, 511, 185

Andreasen, G. K. 1988, A\&A, 196, 159

Antonello, E., \& Poretti, E. 1986, A\&A, 169, 149

Antonello, E., Poretti, E., \& Reduzzi, L. 1990, A\&A, 236, 138

Berdnikov, L. N. 1987, Perem. Zvezdy, 22, 530 1992a, Astron. Astrophys. Trans., 2, 1 1992b, Astron. Astrophys. Trans., 2, 31 1992c, Astron. Astrophys. Trans., 2, 43 1992d, Astron. Astrophys. Trans., 2, 107 1992e, Astron. Astrophys. Trans., 2, 157 1992f, Pis'ma Astron. Zh., 18, 325 1993a, Pis'ma Astron. Zh., 19, 210 1993b, Inf. Bull. Variable Stars, 3863, 1

Berdnikov, L. N., \& Turner, D. G. 1995, Pis'ma Astron. Zh., 21, 803

Berdnikov, L. N., \& Vozyakova, O. V. 1995, Pis'ma Astron. Zh., 21, 348

Diethelm, R. 1983, A\&A, 124, 108 1990, A\&A, 239, 186

Fernie, J. D., \& Ehlers, P. 1999, AJ, 117, 1563

Gingold, R. A. 1976, ApJ, 204, 116

Harris, H. C. 1980, Ph.D. thesis, Univ. Washington 1985, AJ, 90, 756

Harris, H. C., \& Welch, D. L. 1989, AJ, 98, 981

Henden, A. A. 1980, MNRAS, 192, 621 1996a, AJ, 111, 902 1996b, AJ, 112, 2757

Kholopov, P. N. 1985, General Catalogue of Variable Stars, Vols. 1 and 2 (4th ed.; Moscow: Nauka) Nauka)

\section{REFERENCES}

Loomis, C., Schmidt, E. G., \& Simon, N. R. 1988, MNRAS, 235, 1059

Mantegazza, L., \& Poretti, E. 1992, A\&A, 261, 137

Moffett, T. J., \& Barnes, T. F. 1984, ApJS, 55, 389 1985, ApJS, 58, 843

Pel, J. W. 1976, A\&AS, 24, 413

Petersen, O. J. 1986, A\&A, 170, 59

Poretti, E., \& Pardo, I. 1997, A\&A, 324, 133

Schmidt, E. G., Chab, J. R., \& Reiswig, D. E. 1995, AJ, 109, 1239

Schmidt, E. G., Johnston, D., Langan, S., \& Lee, K. M. 2004a, AJ, 128, 1748 (Paper I) 2005, AJ, 129, 2007 (Paper II)

Schmidt, E. G., Johnston, D., Lee, K. M., Langan, S., Newman, P. R., \& Snedden, S. A. 2004b, AJ, 128, 2988

Schmidt, E. G., Lanagan, S., Lee, K. M., Johnston, D., Newman, P. R., \& Snedden, S. A. 2003a, AJ, 126, 2495

Schmidt, E. G., Lee, K. M., Johnston, D., Newman, P. R., \& Snedden, S. A. 2003b, AJ, 126, 906

Schmidt, E. G., \& Reiswig, D. E. 1993, AJ, 106, 2429

Simon, N. R., \& Lee, A. S. 1981, ApJ, 248, 291

Simon, N. R., \& Moffett, T. J. 1985, PASP, 97, 1078

Smith, H. A., Silbermann, N. A., Baird, S. R., \& Graham, J. A. 1992, AJ, 104, 1430

Szabados, L. 1977, Mitt. Sternw. Ung. Akad. Wiss., 70, 1 1980, Comm. Konkoly Obs, 76, 1

1991, Comm. Konkoly Obs, 96, 1

Udalski, A., Soszynski, I., Szymanski, M., Kubiak, M., Pietrzynski, G., Wozniak, P., \& Zebrun, K. 1999a, Acta Astron., 49, 1 1999b, Acta Astron., 49, 437

Zakrzewski, B., Ogloza, W., \& Moskalik, P. 2000, Act. Astron. 50, 387 